\title{
Seguimiento neurofisiológico en injertos de nervios periféricos
}

\author{
ROBERTO PORTILLO ${ }^{1}$, EDGARD ROJAS ${ }^{2}$, JOSÉ VERA², GINA CONCHA ${ }^{2}$ \\ 1) efe de Servicio de N eurología. ${ }^{2}$ M édicos asistentes del Hospital Guillermo Almenara. Essalud Lima- Perú.
}

\section{RESUMEN}

OBJETIVO: Seguimiento neurofisiológico en 10 pacientes con injertos de nervios periféricos. LU GA R: U nidad de N eurofisiología Clínica del H ospital N acional Guillermo A Imenara Irigoyen, EsSalud. MATERIAL Y MÉTODOS: Se estudió 10 pacientes de 6 a 39 años, con lesiones de nervios periféricos en miembros superiores, en quienes se realizó injertos. Se hizo control neurofisiológico periódico con el ectromiografía, velocidad de conducción nerviosa motora y sensitiva. RESULTADO: Se encontró una excelente evolución de los 10 casos, demostrada por el seguimiento electrofisiológico, en algunos de ellos con recuperación precoz de la conducción nerviosa motora e incluso sensitiva, como en los casos de lesión cubital (casos 4, 7 y 8) y cubital y mediano (caso 10). Esta recuperación tuvo un correlato clínico, con cambios neurovegetativos, motores y sensitivos. CONCLUSIONES: La electrofisiología juega un papel importante en el seguimiento postoperatorio de las lesiones de los nervios periféricos en las que se realiza injertos. El tiempo de recuperación neurofisiológica y clínica de un injerto nervioso es muy variable, entre 1 y 2 años o más. Sorprende que la fisiología clínica no siempre guarde una relación proporcional con la electrofisiología, pues en algunos casos la recuperación clínica es aceptable, mientras que en otros es desproporcionada a la gran lentitud de la velocidad de conducción nerviosa.

Palabras clave: Neurofisiología; nervios periféricos, cirugía; fibras nerviosas.

\section{NE UROPHYSIOLOGICAL FOLLOW-UP IN PERIPHERAL NERVES GRAFTS SUMMARY}

OBJECTIVE: Neurophysiologic follow-up of 10 patients with peripheral nerves grafts. SETTING: Clinical Neurophysiology U nit, Guillermo AImenara Irigoyen N ational Hospital, EsSalud. MATERIAL AND METHODS: Ten 6 to 39 year-old patients with nerve grafts for peripheral nerve wounds in upper limbs were studied. Neurophysiologic control by electromyography, motor and sensitive speed nerve conduction was done periodically. RE SU LTS: A $n$ excellent evolution of the 10 peripheral nerve graft cases was demonstrated by the electrophysiology follow up, most of them with early recuperation of the motor nerve conduction and sensitive recuperation, as in cases of cubital lesion (cases 4, 7 and 8) and cubital and median lesions (case 10). Clinical recovery showed correlation with neurovegetative, motor and sensitive changes. CONCLUSIONS: Electrophysiology plays an important role in the evaluation of post-surgery peripheral nerve grafts. The time of clinical and electrophysiolgical recuperation varies from 1 to 2 years or more. Surprisingly, clinical recovery is not always related to electrophysiological findings; in some cases recovery is acceptable and in others the electrophysiological recuperation is slower than clinical recovery.

Key words: Neurophysiology; peripheral nerves, surgery; nerve fibers.

\footnotetext{
Correspondencia:

Dr. Roberto Portillo Vallenas

Hospital Nacional Guillermo Almenara Irigoyen

Unidad de Neurofisiología

Av. Grau 800. Lima 1, Perú

E-mail: drrobertoportillo@ hotmail.com
} 


\section{INTRODUCCIÓN}

Los avances y conocimientos actuales en la microcirugía de los nervios periféricos, han modificado sustancialmente los dogmas que se tenía como inamovibles en las lesiones de los nervios periféricos; así, las más completas parálisis, impresionantes atrofias con pérdida completa de la sensibilidad y de los trastornos neurovegetativos eran consideradas irreversibles e irreparables.

Se ideó diversos métodos para atenuar los trastornos funcionales que tal es lesiones producían, sin tocar la raíz misma del problema, es decir, la reparación del nervio periférico, que actual mente se hace de rutina con bastante éxito $\left({ }^{1,2}\right)$. La electrofisiología clínica constituye en este campo un elemento de importancia y es insustituible en el estudio de la determinación del nivel de la lesión, en la evolución de los casos operados, en el pronóstico y en la evaluación anatómica y funcional de las lesiones de los nervios periféricos ( $\left.{ }^{3-8}\right)$.

A propósito, se presenta una casuística de diez pacientes con injertos de nervios periféricos, que fueron estudiados y operados en los servicios de $\mathrm{N}$ eurología y Cirugía Plástica del Hospital Nacional Guillermo A Imenara Irigoyen, a quienes se realizó un seguimiento clínico y electrofisiológico posquirúrgico.

\section{MATERIAL Y MÉTODOS}

Se estudió diez pacientes con lesiones de nervios periféricos en quienes se practicó evaluación clínica y electrofisiológica después de la cirugía de injertos de nervios periféricos.

En la evaluación del paciente se consignó la filiación, número de seguro, edad, sexo, procedencia, agente causal, nivel y tipo de lesión y el tiempo transcurrido de la lesión.

Para los estudios electrofisiológicos, se empleó un electromiógrafo marca N IKON K OHDEN, electrodos de aguja coaxial para electromiografía
(EMG) y velocidad de conducción nerviosa motora y electrodos de anillos para determinar el potencial sensitivo antidrómico.

L uego se procedió a registrar los resultados de los estudios de electromiografía y velocidad de conducción nerviosa en los pacientes estudiados en una ficha de recolección de datos, de manera de ser analizados y discutidos.

\section{RESULTADOS}

En la Tabla que sigue a continuación se expresa las principales características de los pacientes y el tipo de lesiones que presentaban.

\section{DISCUSIÓN}

E n nuestra casuística, la edad de los pacientes varió de 6 a 39 años, y predominó el sexo masculino en ocho casos. En cuanto a la procedencia, 5 fueron de Lima y 5 de provincia. Los agentes causales fueron elementos cortantes: vidrio, hoja de afeitar, sierras circulares, entre otros.

EI nivel de la lesión de mayor frecuencia fue la muñeca y a predominio derecho, siguiendo en orden el tercio inferior del brazo, palma de la mano y región distal del antebrazo.

Con relación al nivel de la lesión, los nervios más comprometidos fueron el cubital en cinco casos, mediano en dos, y mixto (mediano y cubital) en tres casos; todos tenían lesión total, a excepción de un nervio.

El tiempo entre el accidente y la operación varió de un día a catorce meses. Dos casos fueron reintervenidos. Como nervio dador fue empleado el sural en todos los casos; la longitud del injerto fluctuó entre 2 a 10 centímetros y se empleó de dos a ocho fascículos nerviosos.

L os síntomas fundamentales fueron parálisis y pérdida de la sensibilidad en los diez casos y neuroma en nueve casos. 
Tabla.- Características de los pacientes con lesiones de nervios periféricos.

\begin{tabular}{|c|c|c|c|c|c|c|}
\hline $\mathrm{N}^{\mathrm{o}}$ & Edad & Sexo & Procedencia & Agente causal & Nivel de lesión & Tipo de lesión \\
\hline 1 & 26 & M & Chincha & Vidrio & $\begin{array}{l}\text { Tercio inferior } \\
\text { antebrazo derecho }\end{array}$ & $\begin{array}{l}\text { Lesión total de } \\
\text { nervio cubital }\end{array}$ \\
\hline 2 & 23 & M & Arequipa & Vidrio & Muñeca derecha & $\begin{array}{l}\text { Lesión total de } \\
\text { nervio cubital }\end{array}$ \\
\hline 3 & 19 & M & Lima & $\begin{array}{l}\text { Atricción } \\
\text { por rodillo }\end{array}$ & $\begin{array}{l}\text { Palma de mano } \\
\text { derecha }\end{array}$ & $\begin{array}{l}\text { Lesión total nervio } \\
\text { mediano y cubital }\end{array}$ \\
\hline 4 & 20 & M & Lima & Vidrio & Muñeca derecha & $\begin{array}{l}\text { Lesión total nervio } \\
\text { mediano y cubital }\end{array}$ \\
\hline 5 & 21 & $\mathbf{M}$ & Lima & $\begin{array}{l}\text { Hoja de } \\
\text { de afeitar }\end{array}$ & $\begin{array}{l}\text { Tercio inferior } \\
\text { brazo derecho }\end{array}$ & $\begin{array}{l}\text { Lesión completa } \\
\text { de cubital }\end{array}$ \\
\hline 6 & 39 & M & Lima & $\begin{array}{l}\text { Abrasión } \\
\text { por rodillo }\end{array}$ & Muñeca derecha & $\begin{array}{l}\text { Lesión completa } \\
\text { de mediano }\end{array}$ \\
\hline 7 & 37 & M & Piura & $\begin{array}{l}\text { Atricción } \\
\text { por joya }\end{array}$ & $\begin{array}{l}\text { Tercio inferior } \\
\text { brazo izquierdo }\end{array}$ & $\begin{array}{l}\text { Lesión completa } \\
\text { nervio cubital }\end{array}$ \\
\hline 8 & 23 & M & Lima & Sierra circular & $\begin{array}{l}\text { Muñeca izquierda } \\
\text { transcarpiana }\end{array}$ & $\begin{array}{l}\text { Lesión total nervio } \\
\text { mediano, cubital y } \\
\text { rama } 1^{\text {er }} \text { interóseo } \\
\text { dorsal, parcial } \\
\text { abductor meñique }\end{array}$ \\
\hline 9 & 34 & $\mathrm{~F}$ & Trujillo & $\begin{array}{l}\text { Atricción en } \\
\text { puerta auto }\end{array}$ & $\begin{array}{l}\text { Tercio inferior } \\
\text { antebrazo derecho }\end{array}$ & $\begin{array}{l}\text { Lesión total } \\
\text { nervio cubital }\end{array}$ \\
\hline 10 & 06 & $\mathrm{~F}$ & Chincha & Vidrio & $\begin{array}{l}\text { Región palmar } \\
\text { derecha }\end{array}$ & $\begin{array}{l}\text { Lesión total nervio } \\
\text { mediano y cubital. }\end{array}$ \\
\hline
\end{tabular}

Enseguida se describe la evolución electrofisiológica de nuestros casos.

\section{CASO 1}

Paciente de 26 años con lesión total del nervio cubital derecho a nivel del tercio inferior del antebrazo, producida por herida cortante, con un vidrio el 13 de diciembre de 1990. El primer estudio el ectrofisiológico se realizó a los 4 meses del accidente, encontrándose EM G con denervación total desde el abductor del meñique hasta el primer interóseo dorsal e inexcitabilidad del nervio cubital derecho en el sitio de la lesión. La operación fue el 4 de mayo de 1991.
El segundo control el ectrofisiológico se efectuó a los cinco meses de la operación, encontrándose con las mismas características que el primer estudio. El tercer estudio fue a los 9 meses de operado, observándose en esta ocasión denervación parcial de los músculos comprometidos y en la velocidad de conducción motora se constató que la latencia muñecaabductor del meñique era de $9,7 \mathrm{~m} / \mathrm{seg}$ y al primer interóseo de 18,5 m/seg; la velocidad de conducción codo-muñeca fue $32,5 \mathrm{~m} / \mathrm{seg}$, lo que significa que hay reconexión nerviosa. El cuarto estudio neurofisiológico se realizó a los 18 meses, con EM G normal, latencia normal para muñeca-abductor del meñique y discretamente enlentecida para el primer interóseo, con una 
velocidad de conducción codo-muñeca de 42,5 $\mathrm{m} / \mathrm{seg}$.

A los 26 meses se hizo un último estudio; la velocidad a nivel del injerto fue $19,3 \mathrm{~m} / \mathrm{seg}$ y la conducción de codo encima de la cicatriz $74 \mathrm{~m} /$ seg. Lo que llamó la atención fue la inexcitabilidad del potencial sensitivo antidrómico. Clínicamente, el paciente recuperó la motilidad, el trofismo, la función neurovegetativa, sensibilidad al calor y al frío.

\section{CASO 2}

Paciente de 23 años con lesión total del nervio cubital derecho a nivel de la muñeca, por herida cortante con vidrio el 26 de mayo de 1992.

El primer estudio electrofisiológico se realizó a los 8 meses del accidente, constatándose denervación completa del cuarto lumbrical y parcial del primer interóseo y abductor del meñique derecho.

La latencia distal de la muñeca al abductor del meñique fue $7,1 \mathrm{~m} / \mathrm{seg}$, y al primer interóseo de 10,4 m/seg. La velocidad de conducción de codo-muñeca fue $50 \mathrm{~m} / \mathrm{seg}$, el potencial sensitivo antidrómico fue inexcitable. La operación se practicó el 20 de marzo del 1993. El segundo estudio electrofisiológico fue practicado a los 2 años 8 meses de operado. El estudio electromiográfico practicado en el abductor del meñique, primer interóseo y cuarto lumbrical fue normal, la velocidad de conducción nerviosa motora en la latencia muñeca-abductor del meñique fue $3,9 \mathrm{~m} / \mathrm{seg}$, y al primer interóseo $5,4 \mathrm{~m} / \mathrm{seg}$. La velocidad de conducción codomuñeca fue $58 \mathrm{~m} / \mathrm{seg}$, el potencial sensitivo antidrómico persistió inexcitable. También en este caso hubo recuperación de la motilidad, trofismo y sensibilidad al calor y al frío.

\section{CASO 3}

Paciente de 19 años con lesión completa del nervio mediano derecho a nivel de la palma de la mano por atricción con rodillo; el accidente se produjo el 29 de mayo de 1990. La opera- ción se practicó el 26 de noviembre de 1991. En el primer estudio electrofisiológico practicado al año diez días se encontró en la electromiografía del abductor corto del pulgar derecho unidades motoras de bajo voltaje, con patrón de interferencia incompleto. En cuanto a la velocidad del nervio mediano derecho, se constató una latencia distal muñeca-abductor corto del pulgar de $6,9 \mathrm{~m} / \mathrm{seg}$. Subsistió la inexcitabilidad del potencial sensitivo antidrómico. La recuperación de la motilidad y trofismo fue muy buena.

\section{CASO 4}

Paciente varón de 20 años con lesión de los nervios mediano y cubital derechos a nivel de la muñeca por corte con un vidrio; este accidente sucedió el 5 de abril de 1992. El primer estudio electrofisiológico se practicó al mes del accidente. El estudio EMG del abductor corto del pulgar, abductor corto del meñique y primer interóseo dorsal presentó denervación total.

La velocidad de conducción de los nervios mediano y cubitales objetivó inexcitabilidad tanto motora como sensitiva. La operación se practicó el 16 de julio de 1992 ( 3 meses después del accidente).

El segundo estudio electrofisiológico se realizó a los 6 meses de la operación, constatándose en la EM G denervación total del abductor corto del pulgar y primer interóseo y denervación parcial del abductor del meñique; persistió la inexcitabilidad motora y sensitiva del nervio mediano y en el cubital la latencia muñecaabductor del meñique fue $33,6 \mathrm{~m} / \mathrm{seg}$; la velocidad de conducción codo-muñeca fue $39 \mathrm{~m} / \mathrm{seg}$.

El tercer estudio practicado a los 2 años 5 meses de la operación en los mismos músculos, tuvo un patrón de interferencia incompleto; en cuanto a la velocidad de conducción motora en el mediano, su latencia distal fue $7,7 \mathrm{~m} / \mathrm{seg}$ y la velocidad codo-muñeca $33 \mathrm{~m} / \mathrm{seg}$; el potencial sensitivo antidrómico fue $6,5 \mathrm{~m} / \mathrm{seg}$. Hubo buena recuperación de la funcionalidad y trofismo del segmento afectado. 


\section{CASO 5}

Paciente de 21 años con lesión completa del nervio cubital derecho a nivel del tercio inferior del brazo, por herida cortante con una hoja de afeitar. Este accidente se produjo el 23 de marzo de 1993, la operación se practicó el día 24 de marzo de 1993.

El primer estudio electrofisiológico se realizó a los 10 meses de la operación. En la EM G del abductor del meñique derecho se encontró un patrón de interferencia pobre. La velocidad de conducción motora del nervio cubital derecho mostró en su latencia muñeca-abductor del meñique $5,7 \mathrm{~m} / \mathrm{seg}$, la velocidad de conducción codo-muñeca fue $37,5 \mathrm{~m} / \mathrm{seg}$; a nivel del injerto fue $36,5 \mathrm{~m} / \mathrm{seg}$ y axila-codo fue $40 \mathrm{~m} / \mathrm{seg}$.

El potencial sensitivo antidrómico fue inexcitable en las dos oportunidades. En este caso, la reconexión nerviosa se produjo a los 10 meses del injerto, lo que denotó buena potencia regeneradora del nervio.

\section{CASO 6}

Se produjo una lesión total del nervio mediano derecho a nivel de la muñeca, por una máquina cortadora de papel, en un paciente de 39 años. El 27 de junio de 1993 se produjo el accidente y el injerto se practicó a los 8 meses.

El primer estudio electrofisiológico se efectuó a los 4 meses de la operación, objetivándose denervación parcial del abductor corto del pulgar derecho. La latencia a nivel de la muñecaabductor corto del pulgar fue $4,2 \mathrm{~m} / \mathrm{seg}$, la velocidad de codo-muñeca $46 \mathrm{~m} / \mathrm{seg}$, el potencial sensitivo antidrómico inexcitable. La segunda evaluación se hizo a los 8 meses; la EM G fue igual. La velocidad de conducción en la latencia muñeca-abductor corto fue $3 \mathrm{~m} / \mathrm{seg}$, la conducción codo-muñeca $45 \mathrm{~m} / \mathrm{seg}$ y hubo ausencia del potencial sensitivo antidrómico.

El último estudio se realizó a los 14 meses; la EM G y la velocidad de conducción nerviosa motora estuvieron normales, pero persistió la inexcitabilidad sensitiva. En este caso nos Ila- ma la atención la precoz recuperación a los 4 meses del injerto.

\section{CASO 7}

Paciente de 37 años de edad con lesión completa del nervio cubital izquierdo a nivel del tercio inferior del brazo, producido por la atricción de una joya, el 4 de julio de 1992.

Su primer estudio electrofisiológico se practicó a los 2 meses del accidente y se constató denervación completa de los músculos cubital anterior, abductor del meñique y primer interóseo dorsal izquierdos, con inexcitabilidad de la conducción nerviosa motora y sensitiva en el nervio cubital izquierdo.

El injerto se practicó a los 6 meses del accidente. El segundo estudio se realizó a los 18 meses después de la operación, encontrándose un patrón de interferencia incompleto, en el abductor del meñique y en el primer interóseo. El estudio del nervio cubital mostró una latencia muñeca-abductor del meñique de $3 \mathrm{~m} / \mathrm{seg}$ y al primer interóseo de 4,2 m/seg; la velocidad de conducción codo-muñeca fue $47,5 \mathrm{~m} / \mathrm{seg}$. El potencial sensitivo antidrómico en la latencia distal fue $5,9 \mathrm{~m} / \mathrm{seg}$ (enlentecido).

La tercera evaluación se practicó a los 24 meses y se encontró el patrón de interferencia casi completo en los mismos músculos, la velocidad del cubital en su latencia distal fue $2,6 \mathrm{~m} /$ seg, la velocidad de conducción de codo-muñeca $57,5 \mathrm{~m} / \mathrm{seg}$, al nivel del injerto $49,5 \mathrm{~m} / \mathrm{seg}$ y axila-tercio inferior del brazo $72 \mathrm{~m} / \mathrm{seg}$. Este caso tiene importancia por lo al to de la lesión y por la recuperación clínica y electrofisiológica, incluso en la parte sensitiva.

\section{CASO 8}

Paciente de 23 años, con lesión total del nervio mediano y cubital a la rama del primer interóseo dorsal y parcial abductor del meñique izquierdo a nivel de la muñeca (transcarpiana), a causa de un accidente con sierra circular acaecida el 19 de noviembre de 1992. 
El primer estudio electrofisiológico se realizó a los 7 meses del accidente, constatándose en la EM G denervación total del abductor corto del pulgar y el primer interóseo dosal y parcial del abductor del meñique izquierdo.

$L$ a velocidad de conducción del nervio mediano izquierdo en su latencia muñeca-abductor corto del pulgar fue inexcitable; igualmente se objetivó inexcitabilidad en el nervio cubital del mismo lado en su latencia muñeca-primer interóseo. La latencia muñeca-abductor del meñique fue $4,5 \mathrm{~m} / \mathrm{seg}$, la velocidad de conducción del codo-muñeca del meñique $46 \mathrm{~m} / \mathrm{seg}$. El potencial sensitivo antidrómico en su latencia distal de ambos nervios fue inexcitable. EI injerto se practicó a los 12 meses del accidente. El segundo estudio electrofisiológico se realizó a los 15 meses de la operación, evidenciándose en la EMG denervación parcial del abductor corto del pulgar, abductor del meñique y primer interóseo dorsal izquierdos. En cuanto a la velocidad de conducción en el nervio mediano se constató una latencia distal de $6,6 \mathrm{~m} / \mathrm{seg}$, su velocidad de conducción codo-muñeca fue de $40 \mathrm{~m} / \mathrm{seg}$, el potencial sensitivo antidrómico persistió inexcitable. En cuanto al nervio cubital, en la latencia muñeca-primer interóseo fue 8,2 mseg, la velocidad de conducción codo-muñeca $48,5 \mathrm{~m} / \mathrm{seg}$, la latencia muñeca-abductor del meñique 4,4 m/seg, la velocidad de conducción codo-muñeca $55 \mathrm{~m} / \mathrm{seg}$, el potencial sensitivo antidrómico en su latencia distal $6,7 \mathrm{~m} / \mathrm{seg}$. Es evidente la recuperación clínica y electrofisiológica de los nervios lesionados en el transcurso de 15 meses.

\section{CASO 9}

Paciente femenina de 34 años que el 15 de diciembre de 1992 sufrió lesión total del nervio cubital derecho a nivel de la muñeca, región interna, por atricción a causa del cierre brusco de la puerta de un automóvil. A los 4 meses se realiza el injerto. El estudio el ectrofisiológico ef ectuado a los 3 años de la operación objetivó unidades motoras de bajo voltaje con patrón de interferencia pobre en el abductor del meñique y en el primer interóseo dorsal derechos. La velocidad de conducción en el nervio cubital derecho a nivel del injerto segmento cicatriz de antebrazo- muñeca $(10 \mathrm{~cm})$ fue $25,6 \mathrm{~m} / \mathrm{seg}$, segmento codo-encima de la cicatriz $(10 \mathrm{~cm}) 47,7$ $\mathrm{m} / \mathrm{seg}$. La latencia muñeca-abductor del meñique fue normal $(2,9 \mathrm{~m} / \mathrm{seg})$, el potencial sensitivo antidrómico fue excitable. En este caso nos llamó la atención la presencia de hipotrofia muscular, a pesar de la buena conducción nerviosa, la que se explicaría por la fal ta de ejercicio de la paciente por miedo a la ruptura del injerto.

\section{CASO 10}

Se trata de una niña de 6 años de edad, con lesión total de los nervios mediano y cubital derechos en la región palmar; el accidente se produjo por corte de vidrio el 20 de abril de 1990; la operación se realizó a los 4 meses. Se practicó un estudio electrofisiológico a los 28 meses de la operación. L a electromiografía mostró denervación parcial en el abductor corto del pulgar; la velocidad de conducción en el nervio mediano en su latencia distal (muñeca-abductor del pulgar) fue $5,3 \mathrm{~m} / \mathrm{seg}$ y el potencial sensitivo antidrómico en su latencia distal fue $5,9 \mathrm{~m} /$ seg. El nervio cubital derecho en la latencia muñeca-abductor del meñique fue $3,6 \mathrm{~m} / \mathrm{seg}$, la velocidad de conducción codo-muñeca $57 \mathrm{~m} / \mathrm{seg}$ y el potencial sensitivo antidrómico en la latencia muñeca-dedo meñique $1,9 \mathrm{~m} / \mathrm{seg}$.

En este caso, la recuperación clínica y electrofisiológica fue total, jugando un papel importante la edad de la niña, donde se evidencia una gran regeneración de los nervios periféricos.

\section{COMENTARIO}

La excelente evolución de los 10 casos de injertos de nervios periféricos fue demostrado por el seguimiento electrofisiológico; algunos tuvieron recuperación incluso de la conducción nerviosa sensitiva (casos números 4, 7,8 y 10 ). 
E ste hecho se manifiesta con mayor claridad en el caso número 10, correspondiente a una niña, en quien la recuperación fue completa. Estos datos se corroboran con otros estudios en los cuales se menciona a la edad como un factor importante en la rápida recuperación posquirúrgica en los injertos de nervios periféricos ( $\left.{ }^{8-11}\right)$. Esta recuperación tiene su correlato clínico en una secuencia que se inicia con cambios en el aspecto neurovegetativo, luego motor y sensitivo, casi paralelos. Hemos observado que, en la medida que va desapareciendo la resequedad de la piel en el territorio del nervio seccionado, comienza en forma progresiva, de la región proximal hacia la distal, a aparecer la secreción sudoral y la frialdad de los dedos va adquiriendo una temperatura cerca de lo normal.

O tro hecho que llama la atención es la reaparición de la capacidad discriminativa del frío y el calor, siguiendo luego la sensibilidad táctil, que es recepcionada inicialmente como parestesia, hasta tener sensación dolorosa.

$L$ a sensibilidad gnóstica de discriminación de dos puntos se recupera al final, paralelo a la recuperación sensitiva; la recuperación motora es más precoz, siendo ella más notoria en los músculos proximales y de mayor masa muscular, cualquiera sea el nivel de la lesión y el tiempo de operación, bastando una conducción nerviosa motora de 1 a $5 \mathrm{~m} / \mathrm{seg}$ para adquirir funcionalidad del segmento afectado.

Estos hallazgos concuerdan con los datos encontrados por otros investigadores, quienes mencionan que la recuperación motora, tanto desde el punto de vista clínico como electrofisiológico, se da con anterioridad a la recuperación sensitiva $\left({ }^{9,10}\right)$.

Podemos concluir que, el desarrollo de la microcirugía en todos los campos - traumática, congénita, médica, etc-, está obteniendo logros extraordinariamente sorprendentes, en el sistema nervioso periférico y pares craneales, inclusive. En la microcirugía de los nervios periféricos, la neurofisiología clínica tiene un extenso campo de colaboración, evaluación, determinación, orientación e investigación postoperatoria de primerísima importancia.

D e una pequeña y seleccionada casuística de injertos de nervios periféricos se puede obtener algunas importantes sugerencias:

a) El tiempo de inicio de las primeras manifestaciones electrofisiológicas y por lo tanto clínicas de recuperación funcional del injerto nervioso es muy variable, pudiendo ser hasta de uno o dos años. M ientras tanto, no debe darse por fracasada una operación de este tipo, influyendo factores individuales, cuyos parámetros serán establecidos por la investigación futura. Esto no descarta que se deba intentar la reparación quirúrgica con injertos cuantas veces sea necesario.

b) Lo mismo parece suceder en cuanto al tiempo de la lesión y el de la intervención quirúrgica, que puede ser a corto, mediano o largo plazo sin desfavorecer los resultados; aquí existe un campo amplio para su investigación.

c) Sorprende que la clínica no siempre guarde relación proporcional con la electrofisiología; mientras que en algunos casos esta relación es aceptable y concordante, en otras, la recuperación clínica es completamente desproporcionada con la gran lentitud de la velocidad de conducción nerviosa, por lo que volvemos a insistir en que se debe valorar factores individuales en cada caso particular.

d) La electrofisiología puede poner en evidencia factores que desfavorecen la evolución clínica y que hagan parecer como fracaso una intervención quirúrgica que en realidad es un éxito, desde el punto de vista clínico.

e) Reiteremos, por último, que el trabajo en equipo es la forma más eficiente para obtener los mejores resultados en la evaluación posquirúrgica de los casos.

\section{B IBLIOG RAFÍA}

1. K urze T. Microtechniques in neurological surgery. Clin N eurosur 1964; 11: 128-37. 
2. Donzelli R, Benvenuti D, Schonauer C, Mariniello G, De Divitiis $\mathbf{E}$. M icrosurgical nervous reconstruction using autografts: a two year follow up. J Neurosurg Sci 1998; 42(2): 79-83.

3. Kimura J. Electrodiagnosis in diseases of nerve and muscle: Principles and practice. Edition 2. Philadelphia: FA Davis Company. 1981.

4. J acobson S, G uth L. A n electrophisiological study of the early stages on peripheric nerve regeneration. Expl Neurol 1965; 11: 48.

5. Rodriguez A, Tester $\mathbf{Y} \mathbf{T}$. Fundamentals of electromyography. Chapter 13. En: Electrodiagnosis and electromyography. 3era Ed. N ew Haven C onnecticut: Licht. S 297-351.

6. Baba M, Gillat R, Jacobs J. Recovery of distal changes after nerve constriction by a ligadure. J Neurol Sci 1983; 60: $235-46$.
7. Clough J F M, K unell D, Phillips C G . Conduction velocity in proximal and distal portions of fine limb axons in the baboon. J Physiol 1968; 198: 167-78.

8. Hodes R, Lannabee M G, German W. The human electromyogram in response to nerve stimulation and the conduction velocity of motor axons. Studies on normal and injured peripheral nerves. Arch Neurol Psychiatry 1948; 60: 340-65.

9. Almquist MD, Oloffson $\mathbf{0}$. Sensory nerve conduction velocity and two point discrimination in sutured nerves. J Bone Joint Surg 1970; 52: 791-6.

10. Hafiz $\mathbf{M}$, L ee $\mathbf{S}$, W olf $\mathbf{P}$, Haghighi $\mathbf{P}$. A comparative study of autologous peripheral nerve grafts. M icrosurgery 1985; 6 (3): 135-40.

11. Kolomiri D, Soucacos PN, Beris AE. Nerve grafting in peripheral nerve microsurgery of the upper extremity. M icrosurgery 1994; 15: 506-11. 